\title{
$\beta$-Globin mutation detection by tagged single-base extension and hybridization to universal glass and flow-through microarrays
}

\author{
Coline HM van Moorsel ${ }^{1}$, Erwin E van Wijngaarden ${ }^{2}$, Ivo FAC Fokkema ${ }^{3}$, \\ Johan T den Dunnen ${ }^{3}$, Dirk Roos ${ }^{1}$, Rob van Zwieten ${ }^{1}$, Piero C Giordano ${ }^{3}$ \\ and Cornelis L Harteveld ${ }^{*, 3}$
}

\footnotetext{
${ }^{1}$ Sanquin Research at CLB and Landsteiner Laboratory, Academic Medical Centre, University of Amsterdam, Amsterdam, The Netherlands; ${ }^{2}$ PamGene International BV, 's Hertogenbosch, The Netherlands; ${ }^{3}$ Center of Human and Clinical Genetics, Leiden University Medical Center, Leiden, The Netherlands
}

To test the feasibility of developing a diagnostic microarray for a specific disease, we selected all pathogenic changes of the $\beta$-globin gene occurring at a frequency $\geqslant 1 \%$ in the multi-ethnic Dutch population for analysis. A tagged single-base extension (SBE) approach was used to detect 19 different mutations causing $\beta$-thalassemia or abnormal hemoglobins. In the SBE reaction, the primers were elongated at the $3^{\prime}$ site with a fluorescently labeled dideoxyribonucleotide triphosphate (ddNTP) complementary to the mutation, following tag hybridization to a glass or flow-through microarray. We compared the performance of a generic glass array and a porous system, by testing each mutation separately using heterozygous carriers and by screening a cohort of 40 unknown $\beta$-thalassemia carriers and patients. The results were verified by direct sequencing. The microarray system was able to detect 17 $\beta$-globin mutations simultaneously with $>95 \%$ accuracy in a single SBE reaction. The flow-through array performed slightly better (96\%), but the main advantages of the system included real-time data recording and a considerable time saving achieved through a reduced hybridization time. European Journal of Human Genetics (2004) 12, 567-573. doi:10.1038/sj.ejhg.5201192 Published online 7 April 2004

Keywords: microarray; thalassemia; single-base extension(SBE); minisequencing; mutation detection; flow-through array

Introduction

The hemoglobinopathies belong to the world's most common recessive monogenic disorders in humans. These involve the $\alpha$ - and $\beta$-thalassemias characterized by a deficiency in globin protein synthesis, and the structural mutants of the $\beta$-globin chain causing hemoglobin $\mathrm{S}(\mathrm{HbS})$, $\mathrm{C}$ or $\mathrm{E}$. Worldwide more than 180 different mutations

*Correspondence: Dr CL Harteveld, Center of Human and Clinical Genetics, Hemoglobinopathies Laboratory, Sylvius Laboratorium, Wassenaarseweg 72, 2333 AL Leiden, The Netherlands. Tel: + 3171527 6094; Fax: + 3171527 6075; E-mail: c.I.harteveld@lumc.nl

Received 12 December 2003; revised 5 February 2004; accepted 18 February 2004 causing $\beta$-thalassemia and more than $360 \beta$-globin variants are known. ${ }^{1,2}$ Carrier frequencies are particularly high in regions endemic for malaria; however, due to present-day migration patterns, the mutation spectrum in regions without a history of malaria tropica, like in most industrialized North European countries, changed considerably and requires a diagnostic tool, able to combine highthroughput analysis with the capacity to detect a wide spectrum of relatively low frequent mutations. ${ }^{3}$

In general, carrier detection is performed at the biochemical level with standard hematological methods and confirmation by molecular analysis is usually achieved by direct sequencing. Mutation detection on microarrays 
is a promising diagnostic tool for high-throughput applications. The feasibility of the technique is often addressed by selecting randomly a number of SNPs associated with disease genes to demonstrate a wide application range. ${ }^{4-7}$ Few articles, however, focus on optimization of a larger number of SNPs representing the mutation spectrum for a particular disease gene. ${ }^{8}$ Here we set out to develop an array for the diagnosis of $\beta$-globin defects in populations where many different mutations occur.

\section{Single-base extension}

Mutation detection arrays are often based on hybridization of amplified DNA fragments to allele specific oligo (ASO) probes on the array. ${ }^{9,10}$ However, these arrays suffer from a high rate of false-positive identifications and are less accurate compared to a minisequence approach. ${ }^{4}$ Minisequencing is the target-dependent addition by a DNA polymerase of a specific nucleotide to a single primer. ${ }^{11}$ In an array-based approach, these primer extension reactions are either based on the allele-specificity of the primer, or on extension with a single specific dideoxyribonucleotide triphosphate (ddNTP). In the latter approach, termed single-base extension (SBE), the primer is extended by a DNA polymerase with a single labeled ddNTP that is complementary to the nucleotide at the mutation site. ${ }^{11-14}$ Fan et al ${ }^{15}$ introduced the tag-SBE, in which the primers contain a unique tail of 20 nucleotides at the $5^{\prime}$ side, complementary to a probe on a generic oligonucleotide array (Figure 1). The tag-probes are independent of the specific mutations to be analyzed, and thus facilitate the choice of probes with similar hybridization efficiencies. This allows flexibility in optimization independent of the array content.

\section{Flow-through microarrays}

In general, microarrays are spotted on microscope glass slides as a solid support, in which the reaction mixture is added under a cover slide or to a small well created by a silicon overlay. ${ }^{16}$ Hybridization is dependent on diffusion and environmental factors such as temperature, salt concentration and viscosity, which makes the system inefficient and time consuming. Recently, a flow-through microarray system has been developed that eliminates these limitations by using a porous material as a support for the arrays. ${ }^{17}$ Target molecules are forced through interconnected capillary pores of an array by flow, resulting in diffusion distances in the order of $100 \mathrm{~nm}$. The hybridization process is therefore fully reaction-rate-limited and complete within a few minutes, allowing real-life detection by CCD camera. Hybridization stringency can be varied by adding/removing solutions and changing the temperature to obtain optimal sensitivity and specificity. In this project we have tested and compared the applicability of flow-through technology for mutation detection to the results obtained using glass arrays.

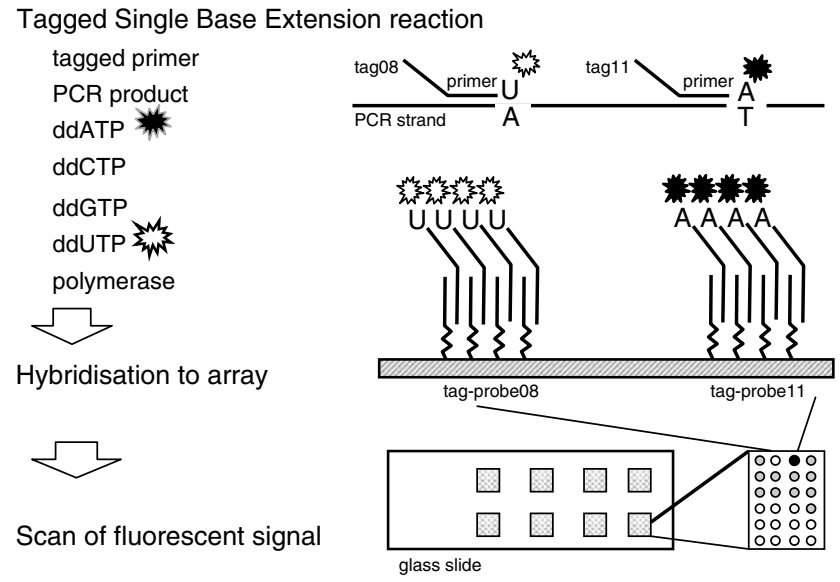

Figure 1 The amplified PCR product is used as a template for the SBE reaction, which is performed in a thermal cycler with a set of 18 mutation-specific primers. Each primer is elongated at the $3^{\prime}$ site with a specific ddNTP, complementary to the wild-type or the mutation. By splitting the SBE reaction into two separate reactions including ddATPCy5/ ddUTP ${ }^{C y} 3 / d d G T P / d d C T P$ and $\operatorname{ddGTP}^{\mathrm{Cy} 5} / \mathrm{ddCTP}^{\mathrm{Cy} 3} / \mathrm{ddATP} /$ ddTTP, all possible combinations can be detected. Each mutation-specific primer contains a unique 20-mer tag at the $5^{\prime}$ site that will hybridize to a spotted complementary sequence on the generic array. A fluorescent signal was detected through scanning and a CCD camera for glass and flow-through microarrays, respectively. Open asterisk, Cy3; closed asterisk, Cy5.

\section{Materials and methods}

Mutation selection and template preparation

A mutation spectrum for $\beta$-thalassemia or $\beta$-globin variant defects was determined from 150 patients and carriers living in The Netherlands, diagnosed over a period of 22 months at the Hemoglobinopathies Laboratory, Leiden. In all, 19 mutations occurred with a frequency $\geqslant 1 \%$. These involved five hemoglobin variants $(\mathrm{HbS}, \mathrm{C}, \mathrm{D}$, $\mathrm{E}$, and $\mathrm{J}$ Baltimore) and $14 \beta$-thalassemia mutations, covering $90 \%$ of the $\beta$-globin mutations in The Netherlands (Figure 2a, Table 1). Iranian DNA samples were obtained from patients treated at the Clinical Center at Bandar Abbas.

DNA was extracted from buffy coats isolated from $5-10 \mathrm{ml}$ whole blood by means of a standard salting-out method $^{18}$ and stored in Tris-HCl EDTA buffer at $4^{\circ} \mathrm{C}$. The $\beta$-globin gene was PCR amplified in two fragments (Figure 2a) ${ }^{19}$ using a Robocycler 96 (Stratagene). In all, $45 \mu \mathrm{l}$ of each PCR product was incubated for $1 \mathrm{~h}$ at $37^{\circ} \mathrm{C}$ with $1.8 \mathrm{U}$ of Shrimp Alkaline Phosphatase (USB) and $18 \mathrm{U}$ of Exonuclease I (USB), inactivated by $15 \mathrm{~min}$ at $97^{\circ} \mathrm{C}$ and purified using QiaQuick columns (Qiagen).

\section{Array preparation and design}

The glass coating and spotting protocol was modified from Beier and Hoheisel $^{20}$ (see www.lgtc.nl). The 20-mer 
a
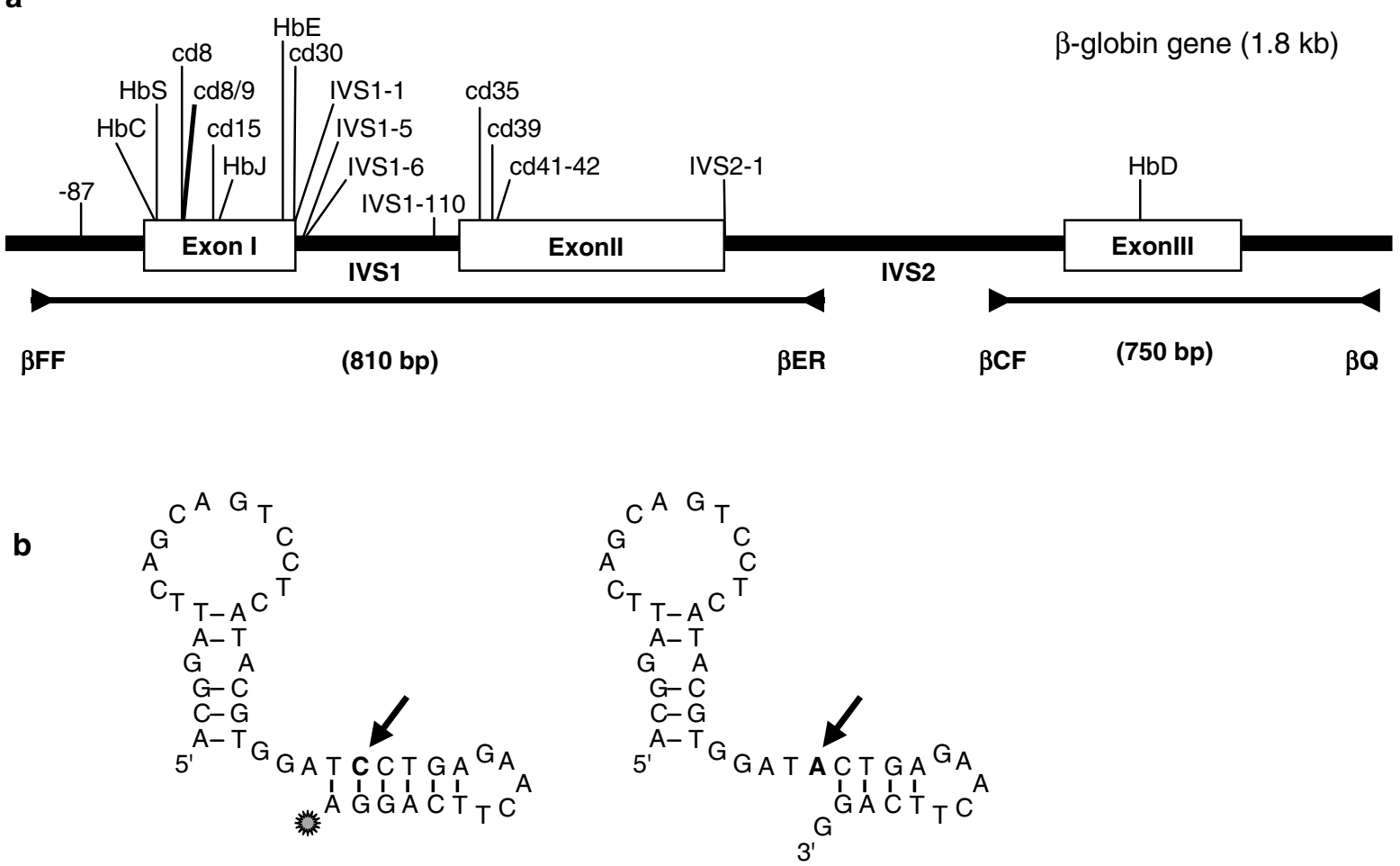

Figure 2 (a) Position of the 19 most frequent mutations within the Dutch population in the $\beta$-globin gene and the position of the PCR primers. Introns in the $\beta$-globin gene are called intervening sequences (IVS). The following primers were used: forward $\beta$ FF 5'-TGAAGTCCAACTCCTAAGCCA, reverse $\beta$ ER 5'-AAACGATCCTGAGACTTCCA, forward $\beta$ CF 5'-GTGTACACATATTGACCAAATC and reverse $\beta Q 5^{\prime}$-CCAAGGTTTGAACTAGCTCTTCA. (b) Secondary structure of the IVS2-1 primer. The first $205^{\prime}$-nucleotides represent the tag, and the last 23 nucleotides are mutation specific. Five consecutive bases at the $3^{\prime}$ terminus are paired. The folded primer incorporates a labeled ddATP in the SBE reaction. The arrows indicate the site of the base substitution applied to prevent self-annealing of the $3^{\prime}$ terminus.

$5^{\prime}-\mathrm{NH}_{2}(\mathrm{C} 6)$ modified oligonucleotide probes (Eurogentec) were spotted with a $417^{\mathrm{TM}}$ Arrayer (Affymetrix). Each array contained 19 probes selected from the universal GenFlex $\AA$ set with a $T_{\mathrm{m}}$ around $58-60^{\circ} \mathrm{C}$ : control set 000-05, 08, 11, $15,17,20,24,27,29,30,33,34,35,36,37,38,41,44,47$ (GenFlex $₫$ Tag Array, Affymetrix P/N 610026). Probe 05 was used as a control to determine the hybridization efficiency by the addition of a $\mathrm{Cy}^{3}$ - and $\mathrm{Cy}^{5}$-labeled oligo with a complementary tag. Per microscope slide, eight arrays were spotted in triplo. The flow-through arrays were PamChip ${ }^{\circledR}$ 's prepared by PamGene (Den Bosch, NL) as previously described, ${ }^{17}$ through spotting of $5^{\prime}$-thiolated oligonucleotides $(0.2 \mathrm{~mm}$ apart $)$ that were covalently coupled to the activated surface. A PamChip ${ }^{\circledR}$ is made of porous $\mathrm{Al}_{2} \mathrm{O}_{3}$ with a pore density of $10^{7} / \mathrm{mm}^{2}$, a pore diameter of $200 \mathrm{~nm}$, and a length of $60000 \mathrm{~nm}$, resulting in a surface area of $500 \mathrm{~cm}^{2} / \mathrm{cm}^{2}$ and an internal volume of $30 \mathrm{nl} / \mathrm{mm}^{2}$ (for details, visit www.pamgene.com). Each PamChip $(\mathbb{R}$ contained four sub-arrays with the same 19

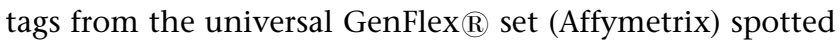
in duplicate.
SBE, hybridization and detection

In all, 18 mutation specific primers (20- to 25-mer) for each of the 19 different mutations were designed for both sense and anti-sense SBE reactions $\left(T_{\mathrm{a}} \approx 61^{\circ} \mathrm{C}\right)$. As primer extension reveals the presence of a mutation by incorporation of a differently labeled ddNTP, a single primer can be used to detect both IVS1-1 mutations. Each mutation-specific primer carried a $5^{\prime}$-tag (20-mer) complementary to a single probe spotted on the array. Primer-tag combinations were checked for secondary structure formation with the DNA Mfold program implemented on the bioinfo.math.rpi.edu/ $\sim$ zukerm site. $^{21}$

The SBE reaction was performed in a total reaction volume of $30 \mu \mathrm{l}$ containing $90 \mathrm{ng}$ PCR-template, 18 different SBE primers $(0.017 \mathrm{pmol} / \mu \mathrm{l}$ each), two labeled ddNTPs $(0.33 \mathrm{pmol} / \mu \mathrm{l}$ each $)$ and two unlabeled ddNTPs $(0.66 \mathrm{pmol} /$ $\mu \mathrm{l}$ each; PerkinElmer Life Sciences Inc.), $6.4 \mathrm{U}$ ThermoSequenase (Amersham Biosciences), in $1 \times$ supplied ThermoSequenase buffer. Two separate SBE reactions per DNA sample were carried out - one containing ddATP-Cy3, ddUTP-Cy5, ddCTP and ddGTP, and another containing 
Table $1 \beta$-mutations with a frequency $\geqslant 1 \%$ in The Netherlands and array results for each mutation

\begin{tabular}{|c|c|c|c|c|c|c|}
\hline \multirow[b]{2}{*}{ Mutation } & \multicolumn{3}{|c|}{ Elongation $^{\mathrm{a}}$} & \multicolumn{3}{|c|}{ Microarray result ${ }^{b}$} \\
\hline & Freq (\%) & SBE WT & SBE Mutant & Hom WT & Het & Hom Mutant \\
\hline$-87(C \rightarrow G)$ & 1 & C & G & + & + & n.a. \\
\hline $\mathrm{HbC}(\mathrm{cd} 6 \mathrm{GAG} \rightarrow \mathrm{AAG})$ & 3 & G & A & + & + & + \\
\hline $\mathrm{HbS}(\mathrm{cd} 6 \mathrm{GAG} \rightarrow \mathrm{GTG})$ & 34 & A & $\mathrm{T}$ & + & + & + \\
\hline $\mathrm{Cd} 8(-\mathrm{AA})$ & 3 & A & G & + & - & n.a. \\
\hline $\mathrm{Cd} 8 / 9(+\mathrm{G})$ & 3 & $\mathrm{~T}$ & G & + & - & n.a. \\
\hline $\mathrm{Cd} 15(\mathrm{TGG} \rightarrow \mathrm{TAG})$ & 1 & G & A & + & + & n.a. \\
\hline $\mathrm{HbJ}$ Baltimore (cd16 GGC $\rightarrow$ GAC) & 1 & G & A & + & + & n.a. \\
\hline $\mathrm{HbE}(\mathrm{cd} 26 \mathrm{GAG} \rightarrow \mathrm{AAG})$ & 8 & G & A & + & $+1-$ & n.a. \\
\hline $\mathrm{Cd} 30(\mathrm{G} \rightarrow \mathrm{C})$ & 1 & G & C & + & + & n.a. \\
\hline IVS1-1 $(G \rightarrow A)$ & 2 & G & A & + & + & n.a. \\
\hline IVS1-1 (G $\rightarrow T)$ & 1 & G & $\mathrm{T}$ & + & + & + \\
\hline IVS1-5 $(\mathrm{G} \rightarrow \mathrm{C})$ & 9 & G & C & + & + & + \\
\hline IVS1-6 (T $\rightarrow C)$ & 2 & $\mathrm{~T}$ & C & + & + & n.a. \\
\hline IVS1-110 (G $\rightarrow A)$ & 7 & G & A & + & + & n.a. \\
\hline Cd $35(-C)$ & 2 & $\mathrm{C}$ & $\mathrm{T}$ & + & + & n.a. \\
\hline $\mathrm{Cd} 39(\mathrm{CAG} \rightarrow \mathrm{TAG})$ & 4 & C & $\mathrm{T}$ & + & $+1-$ & + \\
\hline $\mathrm{Cd} 41-42(-\mathrm{TCTT})$ & 1 & C & G & + & $+1-$ & n.a. \\
\hline IVS2-1 $(G \rightarrow A)$ & 5 & G & A & + & + & + \\
\hline $\mathrm{HbD}(\mathrm{cd} 121 \mathrm{GAA} \rightarrow \mathrm{CAA})$ & 1 & G & C & + & + & n.a. \\
\hline
\end{tabular}

${ }^{a}$ Extension of the mutation-specific primer in an SBE reaction with a wild-type (WT) and a mutant template.

${ }^{\mathrm{b}}$ The microarray results for homozygous (hom.) wild-type, heterozygous (het.) and homozygous mutant: +, detected; -, not detected; n.a., not available in random selection. Results have been obtained from glass and flow-through arrays.

ddATP, ddTTP, ddCTP-Cy3 and ddGTP-Cy5 - to allow the detection of all possible mutations using a two- channel scanner device ( $418^{\mathrm{TM}}$ Scanner, Affymetrix, filters 532 and $635 \mathrm{~nm}$ for Cy3 and Cy5 detection, respectively). The SBE reaction was performed with an initial denaturation step $3.5 \mathrm{~min}$ at $92^{\circ} \mathrm{C}$ and 40 two-step cycles of $45 \mathrm{~s} 92^{\circ} \mathrm{C}$ and $30 \mathrm{~s}$ $61^{\circ} \mathrm{C}$ in Robocycler 96 (Stratagene).

The glass slide was covered with a punched rubber overlay forming eight incubation chambers, one triplo array per incubation chamber, put into a home-made Perspex holder and cover with eight holes for the addition of sample, kept in place by clamps. In all, $10 \mu \mathrm{l}$ of the SBE reaction was added to $50 \mu \mathrm{l}$ of hybridization solution (3.4× SSC, $0.3 \%$ SDS (w/v) in Tris EDTA) and added to a well on the glass array. During hybridization at $40^{\circ} \mathrm{C}$ o.n., the holes were sealed with tape to prevent evaporation. The arrays were washed for $5 \mathrm{~min}$ subsequently in $2 \times$ SSC, $70 \%$ ethanol and $100 \%$ ethanol, and air-dried.

For the PamChip ${ }^{\circledR}$, incubation and detection was performed on a prototype of the FD10 Pam Microarray system (PamGene and Olympus Optical). Incubations were performed in a thermostatically controlled incubator holding one chip, containing four arrays. Prior to hybridization, each array was washed at $40^{\circ} \mathrm{C}$ with respectively $20 \mu \mathrm{l}$ of PBS/0.1\% (w/v) Tween 20 and $20 \mu \mathrm{l}$ of $3 \times$ SSP $(20 \times \mathrm{SSP}=180 \mathrm{mmol} / \mathrm{l} \mathrm{NaCl}$ and $10 \mathrm{mmol} / \mathrm{l}$ Sodium phosphate). Both solutions were pumped one cycle (30s) down and up through the pores of the array using a Microlab 500 syringe pump (Hamilton). A measure of $10 \mu \mathrm{l}$ of the SBE reaction was diluted with $7 \mu$ of $\mathrm{ddH}_{2} \mathrm{O}$, boiled for $5 \mathrm{~min}$ and cooled on ice. After addition of $3 \mu \mathrm{l}$ of $20 \times$ SSP, the solution was hybridized for $5 \mathrm{~min}$ at $40^{\circ} \mathrm{C}$. The solution was pumped down, and images were captured with an 8-bit CCD camera.

All arrays were analyzed using ArrayPro (Media Cybernetics). The spot intensity was calculated after reduction of the median spot intensity with the median local background intensity. The signal was scored positive when it was found at least twice in the same array with an intensity above 5 on a scale of $0-255$.

\section{Results}

\section{SBE optimization}

In all, 18 sense and anti-sense primers were designed to detect 19 mutations and tested individually on a wild-type template. Nonspecific or no signal was observed in seven sense and eight anti-sense primers. We have chosen to optimize the sense primers, except for the anti-sense Cd121, which performed as required (Table 2). For the primers used to detect the Cd16, IVS2-1 and Cd39 mutations, a false-positive signal was observed even in the absence of the template. Analysis in DNA Mfold ${ }^{21}$ predicted the formation of a stable stem loop with three to five consecutive base pairings at the $3^{\prime}$ end (Figure $2 b$ ), which could explain the auto-extension signal during SBE. New primers were designed containing an internal mutation to prevent self-annealing at the $3^{\prime}$ terminus for Cd16, IVS2-1 and Cd39. The modified primers showed an increase in specificity by the loss of auto-extension, but 
Table 2 Mutation specific primers

\begin{tabular}{lrl}
\hline Mutation & $\begin{array}{l}\text { GenFlex }^{\circledR} \text { tag } \\
\text { control set }\end{array}$ & SBE primer sequence $\left(5^{\prime} \rightarrow 3^{\prime}\right)$ \\
\hline-87 & 00029 & CCTCACCCTGTGGAGCCACAC \\
$\mathrm{HbC}$ & 00030 & CACCATGGTGCACCTGACTCCT \\
$\mathrm{HbS}$ & 00037 & ACCATGGTGCACCTGACTCCTG \\
Cd 8 & 00036 & GTGCACCTGACTCCTGAGGAG \\
Cd 8/9 & 00020 & GCACCTGACTCCTGAGGAGAAG \\
Cd15 & 00038 & GAAGTCTGCCGTTACTGCCCTGT \\
$\mathrm{HbJ}$ & 00027 & TGCCGTTACTGACCTGTGGG \\
$\mathrm{HbE}$ & 00035 & GGTGAACGTGGATGAAGTTGGTGGT \\
Cd30 & 00017 & TTGGTGTGAGGCCCTGGGCA \\
IVS1-1 & 00044 & GGTGGTGAGGCCCTGGGCAG \\
IVS1-5 & 00034 & GTGAGGCCCTGGGCAGGTTG \\
IVS1-6 & 00011 & GGTGAGGCCCTGGGCAGGTTGG \\
IVS1-110 & 00047 & GGCACTGACTCTCTCTGCCTATT \\
Cd 35 & 00015 & CTTAGGCTGCTGGTGGTCTACC \\
Cd39 & 00008 & CTGGTGGTCTACCCTTGGACC \\
Cd41-42 & 00033 & TCTACCCTTGGACCCAGAGGTT \\
IVS2-1 & 00024 & ACGTGGATACTGAGAACTCAGG \\
HbD & 00041 & GCCTGCACTGGTGGGGTGAATT \\
\hline Alf &
\end{tabular}

All primers were synthesized as forward except for the Cd121 primer, which was synthesized as reverse. The bold nucleotides in the primers for IVS2-1 and Cd16 indicate the modification applied to prevent autoextension.

the signal for Cd39 dropped below the detection level. The original Cd39 primer could still be used in the assay, because the false-positive signal with ddATP did not interfere with the capacity of the primer to distinguish between the wild-type (ddCTP) and mutant (ddTTP) allele.

The effect of primer concentration on signal intensity was tested by using seven different dilutions in a multiplex of four primers, ranging from 5 to $0.125 \mathrm{pmol}$ in a $30 \mu \mathrm{l} \mathrm{SBE}$ reaction (Figure 3a). The signal for primers Cd39, Cd41-42 and IVS1-1 was optimal at $0.5 \mathrm{pmol}$ each, and no optimum was seen for the Cd121 primer. In the final multiplex SBE containing 18 mutation-specific primers, a concentration of $0.5 \mathrm{pmol} / 30 \mu \mathrm{l}$ for each primer was used.

The ability of the system to detect all of the 19 mutations simultaneously in a single SBE reaction was tested. Out of 19 heterozygous carriers, 17 were scored correctly, that is, both the wild-type and the mutant signal were detectable. No mutant signal was observed for Cd8 and Cd8/9, and these primers were omitted from further analysis. For all mutations, we observed a reduction in wild-type signal to half the intensity in the heterozygotes as compared to the homozygous wild-types.

\section{Screening for $\beta$-globin mutations}

The specificity of the system was tested in a blinded screening of 20 selected Dutch and 20 Iranian samples ( $n=80$ alleles) for which the genotypes were confirmed by direct sequencing. These include 23 wild-type and 57 mutant alleles, representing 14 different mutations. Per sample a single SBE reaction with the 17 SBE primers was performed, one-third was used for hybridization to the glass-array and one-third for the flow-through (Figure 3b). Out of 80 alleles, 76 alleles were scored correctly on glass (95\%) and 77 on PamChip ${ }^{\circledR}$ (96\%). A homozygote HbE was once incorrectly scored as a heterozygous on the glass array, while on the flow-through array a heterozygous carrier for HbE was scored as a homozygote. Both on glass and flow-through array a heterozygote for $\mathrm{Cd} 41 / 42$ was scored as wild-type due to low signal, and the compound heterozygote Cd41-42/IVS1-5 was erroneously scored as a combination of homozygosity for mutation Cd41-42 and heterozygosity for IVS1-5. The Cd39 mutation in the compound heterozygote Cd39/IVS2-1 was missed on the glass array.

\section{Discussion}

A microarray system, using tagged SBE and hybridization to generic home-made glass arrays and commercial PamChip ${ }^{\circledR}$, was developed to detect 19 different $\beta$-thalassemia and $\beta$-globin variant mutations simultaneously. The SBE reaction and hybridization conditions to the array were optimized using a wild-type template and the specificity was tested on PCR templates heterozygous for each mutation. Out of 19 mutations, 17 could be detected reproducibly, which cover more than $80 \%$ of the mutation spectrum observed in The Netherlands and other industrialized North European countries with similar mutation spectra due to recent immigration. The specificity of the system was tested on 40 sequenced carriers and patients samples. Out of 80 alleles, the genotype was correctly assigned in 76 using the glass array and 77 with PamChip ${ }^{\circledR}$, reaching a 95\% reliability. As the selected set includes the most frequent Mediterranean, Asian and African mutations, the system is widely applicable for high-throughput diagnostic purposes in many different countries.

Factors that influence signal intensity and specificity are primer concentration, hybridization efficiency and primer sequence. The primer concentration was varied to reach an optimal signal intensity. If the concentration is too low, the signal remains below detection level, while higher amounts are not extended efficiently, causing competition between tags of extended and nonextended primers for the probes on the array resulting in an overall reduction in signal.

In three mutation-specific tag-primers (cd16, IVS2-1 and cd39), a false-positive signal was observed in the absence of template. Template-independent primer extension might be due to the formation of secondary structures. This has occasionally been observed, particularly in assay formats based on immobilized primers. ${ }^{4,14}$ According to Pastinen et $\mathrm{al}^{4}{ }^{4}$ this problem can be avoided by performing the minisequencing reactions at an elevated temperature or by analyzing the other strand of the DNA template. In our 
a

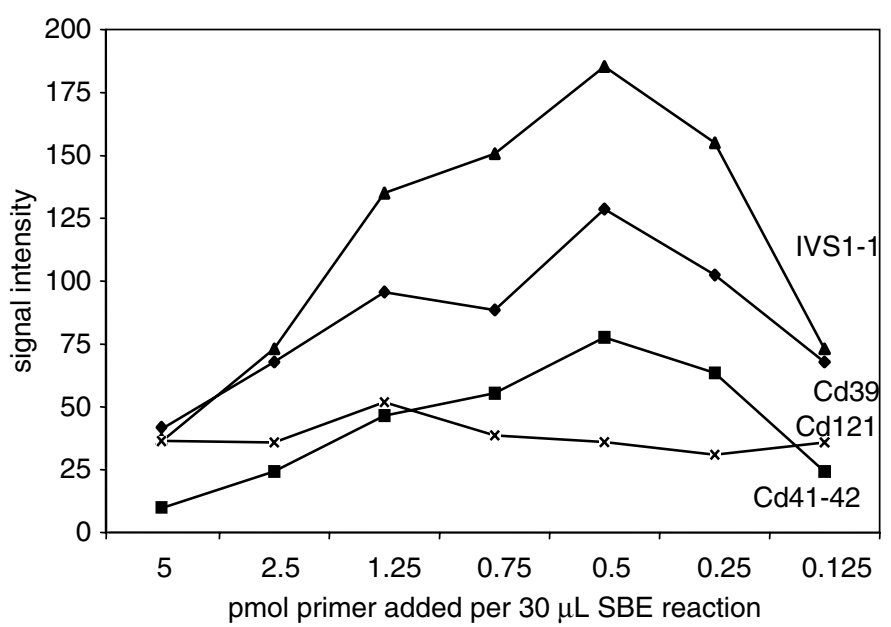

b Glass array
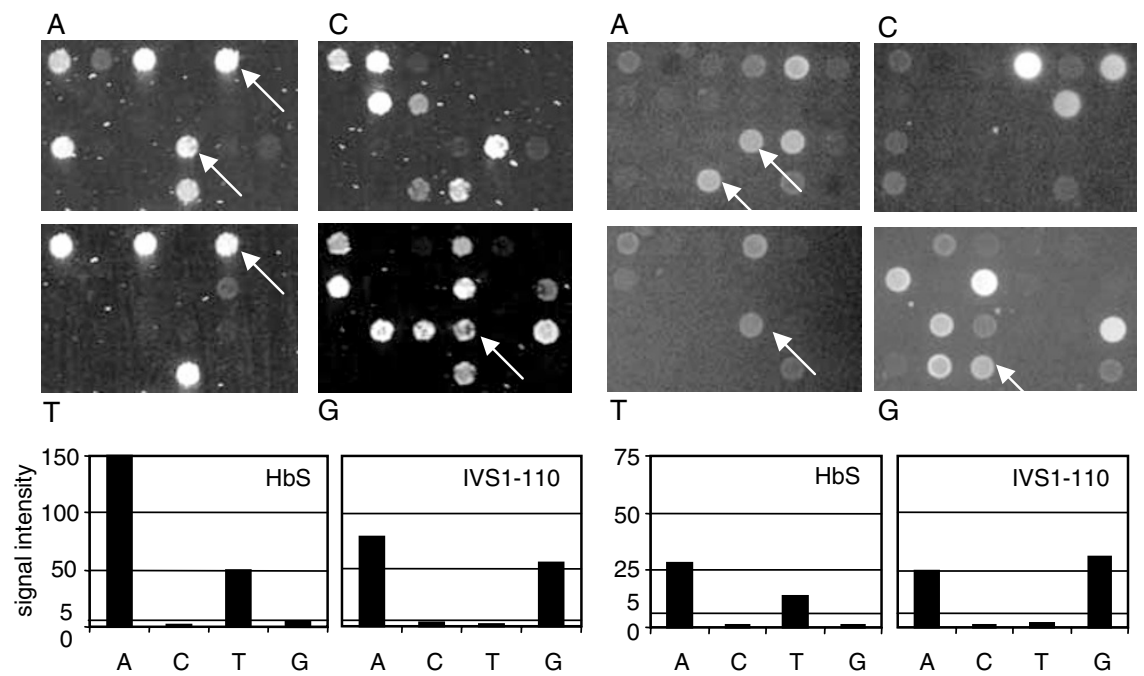

Figure 3 (a) Wild-type signal intensity found with four different primers in seven different concentrations. The SBE reaction was performed on wild-type PCR product and hybridized to a glass array. (b) Scans of glass and flow-through arrays and the corresponding signal after hybridization of a compound heterozygous patient $\mathrm{HbS}(\mathrm{A} \rightarrow \mathrm{T}) / \mathrm{IVS} 1-110(\mathrm{G} \rightarrow \mathrm{A})$. Each array contains four rows and six columns. Marker spots are located on the glass array at the first and fourth row, first and fourth columns. The spots for HbS and IVS1-110 are indicated by arrows. The positions of the probes are different for the glass and flow-through array: on the left, the scans of the glass array for each nucleotide (first row, fifth column HbS spot; third row, fourth column IVS1-110 spot), and on the right the flow-through array (third row, fourth column HbS spot; fourth row, third column IVS1-110 spot). Images of glass arrays were taken after overnight hybridization and subsequent wash; images of the flow-through arrays were taken after 5 min hybridization with subsequent pumping down of the fluid and no wash. In the graphs below, the signal for the HbS and IVS1-110 spot for each possible extension with a ddNTP is given.

study, SBE was performed at a relatively high temperature of $61^{\circ} \mathrm{C}$, which did not prevent the problem. We were able to avoid the false signal in two cases by altering the primer at the base complementary to the $3^{\prime}$ terminus (Figure $2 b$ ).

Out of the 19 mutations tested, three are frameshift mutations involving insertion or deletion of one or several nucleotides. Remarkably, two of these, that is, Cd8(-AA) and $\operatorname{cd} 8 / 9(+\mathrm{G})$, did not show the correct genotype when tested in homo- or heterozygotes, while the
cd41-42(-TCTT) was among the alleles scored erroneously in the blind screening of 80 alleles, due to low mutant signal intensity. It is not clear however, how this relates to secondary structures in the PCR products. Apparently, incorporation efficiencies seem to be largely dependent on the local sequence environment. Syvänen ${ }^{5}$ suggested that the labels might affect the specificity of the nucleotide incorporation by the DNA polymerase. However, overall preference for incorporation of a specific labeled 
nucleotide was not observed among the 17 other primers included in the set.

Other techniques frequently used to detect $\beta$-globin mutations in a diagnostic setting are ASO probe hybridization, ${ }^{22}$ denaturing gel gradient electrophoresis (DGGE), ${ }^{19}$ and amplification refractory mutation system (ARMS). ${ }^{23}$ Most of these approaches are time consuming, require multiple reactions per patient, radioactive reagents or expensive laboratory equipment, and are not always suited for high-throughput analysis. The SBE and hybridization to generic arrays show high specificity (95\%) and flexibility, since no new arrays are needed to change the set of mutations to be analyzed, making the system easily adaptable to changing reasearch goals. As glass coating is performed on microscope slides with relatively simple chemicals and only minimal amounts of probes are used by the $417^{\mathrm{TM}}$ arrayer, arrays can be produced at low cost ( $€ 0.12$ per array). The total price for the SBE reaction is approximately $€ 8$; - per sample, the majority of the expenses involve the enzymes and labeled ddNTPs. This is competitive with direct sequencing of the complete $\beta$-globin gene, as presently used by many diagnostic laboratories.

Our experiments clearly show that the probes can be easily transferred from the glass to the PamChip ${ }^{\circledR}$ microarray system. The main advantage of the flow-through system is the considerably reduced hybridization time, which lasts only $5 \mathrm{~min}$ compared to $16 \mathrm{~h}$ for glass arrays. Additional time is saved by sample preparation and by the real-time recording of hybridization results by the CCD camera, which permits an automated registration and analysis of data. The ability of the system to vary hybridization stringency allows temperature-dependent optimization of mutations, not detectable under normal circumstances. These possibilities will be explored in future studies.

\section{Acknowledgements}

The authors thank Victor de Jager for optimization of the glass-coating protocol and Majid Yavarian for providing the Iranian samples.

\section{References}

1 Weatherall DJ, Clegg JB: The Thalassaemia Syndromes. Oxford: Blackwell Publishing; 1981, (3rd edn).

2 Thein SL: Beta-thalassaemia. Baillières Clin. Haematol. 1998; 11: 91-126.

3 Giordano PC, Harteveld CL, Heister AJGM et al: The molecular spectrum of beta-thalassemia and abnormal hemoglobins in the allochtonous and autochtonous Dutch population. Community Genet 1998; 1: 243-251.

4 Pastinen T, Kurg A, Metspalu A, Peltonen L, Syvänen AC: Minisequencing: a specific tool for DNA analysis and diagnostics on oligonucleotide arrays. Genome Res 1997; 7: 606-614.

5 Syvänen AC: From gels to chips: 'Minisequencing' primer extension for analysis of point mutations and single nucleotide polymorphisms. 1999 [Review]. Hum Mutat 1999; 13: 1-10.

6 Gerry NP, Witowski NE, Day J, Hammer RP, Barany G, Barany F: Universal DNA microarray method for multiplex detection of low abundance point mutations. J Mol Biol 1999; 292: 251-262.

7 Fortina P, Delgrosse K, Sakamuze T et al: Simple two-color arraybased approach for mutation detection. Eur J Hum Genet 2000; 8: 884-894.

8 Gemignani F, Perra C, Landi S et al: Reliable Detection of $\beta$-Thalassemia and G6PD Mutations by a DNA Microarray. Clin Chem 2002; 48: 2051-2054.

9 Hacia JG, Brody LC, Chee MS, Fodor SP, Collins FS: Detection of heterozygous mutations in BRCA1 using high density oligonucleotide arrays and two-colour fluorescence analysis. Nat Genet 1996; 14: 441-447.

10 Wang DG, Fan JB, Siao CJ et al: Large-scale identification, mapping, and genotyping of single-nucleotide polymorphisms in the human genome. Science 1998; 15 280: 1077-1082.

11 Syvänen AC, Aalto-Setälä K, Harju L, Kontula K, Söderlund H: A primer-guided nucleotide incorporation assay in the genotyping of apolipoprotein E. Genomics 1990; 8: 684-692.

12 Sokolov BP: Primer extension technique for the detection of single nucleotides in genomic DNA. Nucleic Acids Res 1990; 18: 3671.

13 Kuppuswami MN, Hoffmann JW, Kasper CK, Spitzer SG, Groce SL: Single-nucleotide primer extension to detect genetic diseases: experimental application to hemophilia B (factor IX) and cystic fibrosis genes. Proc Natl Acad Sci USA 1991; 88: 1143-1147.

14 Nikiforov TT, Rendle RB, Goelet P et al.: Genetic bit analysis: a solid-phase method for typing single nucleotide polymorphisms. Nucleic Acids Res 1994; 22: 4167-4175.

15 Fan J-B, Chen X, Halushka MK et al: Parallel genotyping of human SNPs using generic high-density oligonucleotide tag arrays. Genome Res 2000; 10: 853-860.

16 Pastinen T, Raitio M, Lindroos K, Tainola P, Peltonen L, Syvänen AC: A system for specific, high-throughput genotyping by allelespecific primer extension on microarrays. Genome Res 2000; 10: $1031-1042$.

17 Beuningen $\mathrm{R}$ van, van Damme $\mathrm{H}$, Boender P, Bastiaensen $\mathrm{N}$, Chan A, Kievits T: Fast and specific hybridization using flowthrough microarrays on porous metal oxide. Clin Chem 2001; 47: 1931-1933.

18 Miller SA, Dykes DD, Polesky HF: A simple salting-out procedure for extracting DNA from human nucleated cells. Nucleic Acids Res 1988; 16: 1215

19 Losekoot M, Fodde R, Harteveld CL, van Heeren H, Giordano PC, Bernini LF: Denaturing gradient gel electrophoresis and direct sequencing of PCR amplified genomic DNA: a rapid and reliable diagnostic approach to beta-thalassaemia. Br J Haematol 1990; 76: 269-274.

20 Beier M, Hoheisel JD: Versatile derivatisation of solid support media for covalent bonding on DNA-microchips. Nucleic Acids Res 1999; 27: 1970-1977.

21 SantaLucia Jr J: A unified view of polymer, dumbbell, and oligonucleotide DNA nearest-neighbor thermodynamics. Proc Natl Acad Sci USA. 1998; 95: 1460-1465.

22 Cai SP, Wall J, Kan YW, Chehab FF: Reverse dot blot probes fot the screening of $\beta$-thalassemia mutations in Asians and American Blacks. Hum Mutat 1994; 3: 59-63.

23 Old JM, Varawella NY, Weatherall DJ: Rapid detection and prenatal diagnosis of $\beta$-thalassemia: studies in Indian and Cypriot populations in the UK. Lancet 1990; 336: 834-837. 